\title{
Spectrometer for X-ray emission experiments at FERMI free-electron-laser
}

Cite as: Rev. Sci. Instrum. 85, 103112 (2014); https://doi.org/10.1063/1.4898315

Submitted: 20 June 2014 . Accepted: 01 October 2014 . Published Online: 17 October 2014

L. Poletto, F. Frassetto, P. Miotti, A. Di Cicco, P. Finetti, C. Grazioli, F. lesari, A. Kivimäki, S. Stagira, and M. Coreno

\section{ARTICLES YOU MAY BE INTERESTED IN}

The electronic characterization of biphenylene-Experimental and theoretical insights from core and valence level spectroscopy

The Journal of Chemical Physics 142, 074305 (2015); https://doi.org/10.1063/1.4907723

Modular soft $\mathrm{x}$-ray spectrometer for applications in energy sciences and quantum materials Review of Scientific Instruments 88, 013110 (2017); https://doi.org/10.1063/1.4974356

CITIUS: An infrared-extreme ultraviolet light source for fundamental and applied ultrafast science

Review of Scientific Instruments 85, 023104 (2014); https://doi.org/10.1063/1.4864298

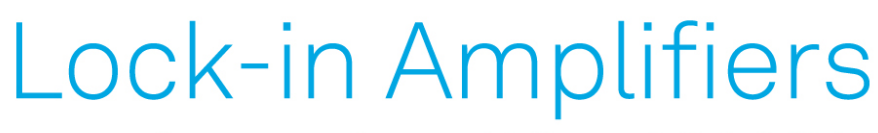
... and more, from DC to $600 \mathrm{MHz}$

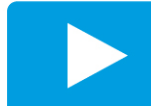

Watch 


\title{
Spectrometer for X-ray emission experiments at FERMI free-electron-laser
}

\author{
L. Poletto, ${ }^{1, a)}$ F. Frassetto, ${ }^{1}$ P. Miotti, ${ }^{1}$ A. Di Cicco, ${ }^{2}$ P. Finetti, ${ }^{3}$ C. Grazioli, ${ }^{4,5}$ F. lesari, ${ }^{2}$ \\ A. Kivimäki, ${ }^{5}$ S. Stagira, ${ }^{6}$ and M. Coreno ${ }^{3,7}$ \\ ${ }^{1}$ CNR - Institute of Photonics and Nanotechnologies (CNR-IFN), via Trasea 7, I-35131 Padova, Italy \\ ${ }^{2}$ Physics Division, School of Science and Technology, Università di Camerino, I-62032 Camerino (MC), Italy \\ ${ }^{3}$ ELETTRA - Sincrotrone Trieste, Basovizza Area Science Park, S. S. 14 - km 163,5, I-34149, \\ Basovizza (TS), Italy \\ ${ }^{4}$ Department of Chemical and Pharmaceutical Sciences, University of Trieste, Via L. Giorgieri 1, \\ I-34127 Trieste, Italy \\ ${ }^{5}$ CNR-Istituto Officina dei Materiali (CNR-IOM), Laboratorio TASC, I-34149 Trieste, Italy \\ ${ }^{6}$ Politecnico di Milano - Department of Physics, I-20133 Milano, Italy \\ ${ }^{7}$ CNR - Istituto di Struttura della Materia (CNR-ISM), UOS Basovizza, I-34149 Trieste, Italy
}

(Received 20 June 2014; accepted 1 October 2014; published online 17 October 2014)

\begin{abstract}
A portable and compact photon spectrometer to be used for photon in-photon out experiments, in particular x-ray emission spectroscopy, is presented. The instrument operates in the $25-800 \mathrm{eV}$ energy range to cover the full emissions of the FEL1 and FEL2 stages of FERMI. The optical design consists of two interchangeable spherical varied-lined-spaced gratings and a CCD detector. Different input sections can be accommodated, with/without an entrance slit and with/without an additional relay mirror, that allow to mount the spectrometer in different end-stations and at variable distances from the target area both at synchrotron and at free-electron-laser beamlines. The characterization on the Gas Phase beamline at ELETTRA Synchrotron (Italy) is presented. () 2014 AIP Publishing LLC. [http://dx.doi.org/10.1063/1.4898315]
\end{abstract}

\section{INTRODUCTION}

X-ray emission spectroscopy (XES) and Resonant Inelastic X-Ray Scattering (RIXS) are standard techniques in surface and solid-state investigations at third generation synchrotron radiation sources. ${ }^{1-4}$ However, the development of 4th generation free-electron-laser (FEL) sources such as FERMI, FLASH, LCLS, and XFEL opens new perspectives for single-shot XES and RIXS measurements of low-density, liquid, and condensed matter. ${ }^{5-9}$ The availability of $10^{12}-10^{14}$ $\mathrm{X}$-ray photons in a single pulse in the sub-ps range allows to study ultrafast single and multi-photon excitation and, by means of pump and probe schemes, excitation dynamics involving core levels. The high photon yield per pulse, achievable in principle with FEL sources, allows also an improvement in the collection of high-quality spectra with a single shot or with a limited number of pulses of selected intensity. All these features open the way to novel applications of XES and RIXS to materials under extreme conditions or to isolated molecules and clusters in the soft and hard Xray regimes. The instrument that is presented here is designed for photon in-photon out experiments at synchrotron and FEL beamlines. In particular, the equipment is intended to be used at the Low-Density-Matter (LDM) ${ }^{10}$ and Elastic and Inelastic Scattering - TIme-resolved studies of Matter under EXtreme and metastable conditions (EIS-TIMEX) ${ }^{11}$ beamlines of FERMI. ${ }^{12}$

In the field of atomic and molecular physics, the use of XES and RIXS techniques is still limited, the main limiting factors being the low density of the sample and the

a) Author to whom correspondence should be addressed. Electronic mail: poletto@dei.unipd.it. small solid angle of collection for photons. Nevertheless new projects are currently developed at the 3rd generation synchrotron machines, taking advantage of the improvement in beamlines and spectrometer performances. At the same time, the complexity of systems whose electronic structure can be investigated in the gas phase with inner-shell photoexcitation and photoionization techniques is continuously increasing. It is now possible to perform experiments on a wide choice of organic molecules of biological interest, ${ }^{13}$ metal-organic complexes,${ }^{14}$ and clusters. ${ }^{15}$ Given the extremely low number of target particles in typical experiments, it is expected that inner-shell studies of clusters would greatly benefit from the advances in the FEL radiation sources. Indeed, an innershell photoionization study of rare-gas clusters was among the first experiments performed at the first European FEL facility (TESLA, Hamburg). ${ }^{16}$ Photon in-photon out experiments are foreseen at FERMI, its low-photon energy $(25-100 \mathrm{eV})$ and in its high photon-energy $(100-800 \mathrm{eV})$ configurations, called as FEL-1 and FEL-2, respectively. In particular access will be gained not only to the $1 \mathrm{~s}$ edges of light elements such as carbon, nitrogen, and oxygen but also to L-edges of most 3d metals. Additional perspectives in RIXS schemes are added when considering pump-probe experiments. When using an optical laser photon as a pump, the probing of core electrons will allow a direct investigation of photophysical and photochemical processes with the elemental sensitivity of core electron spectroscopies. Finally, experimental applications of the apparatus concern time-resolved pump-probe experiments in the strong-optical-field regime, since such experiments will exploit the combination of an atomic/molecular gas jet, FEL radiation and synchronized ultrashort and intense optical laser pulses. 
Various combination of photon-in photon-out spectroscopies have been used for chemical and structural investigations of condensed matter. ${ }^{17}$ The availability of an efficient detector for XES would help the deeper understanding of ultrafast transitions occurring in solid targets (surfaces and thin films) as an effect of the interaction with sub-picosecond pulses. The simplest configuration that can be adopted in a typical pilot experiment using FEL radiation is to use the beam as a pump to produce excited states up to the warm dense matter regime (see Ref. 18 and references therein). Simple calculations show that extreme high pressure and temperature regimes are obtained and kept, at solid-state densities, for a few picoseconds after the pulse. The X-ray emission will be then that characteristic of the strongly excited system and new information about the electron and atomic structure can be obtained by these challenging single-shot measurements. We expect that for high intensities ( $>10^{12}$ photons/pulse), measurements with reasonable statistics collecting a few FEL excitation events are obtained. Typical experiments include simple ultra-thin foils and/or surfaces of light elements and compounds ( $\mathrm{Si}, \mathrm{Al}$, and their oxides) and light metals ( $\mathrm{Li}, \mathrm{Na}$, and $\mathrm{Mg}$ ) for which electron excitation energies are found in the energy range of FEL-1. Those experiments can be extended to $\mathrm{Be}, \mathrm{B}$, and $\mathrm{C}$ using the FEL-2 source. An important advantage of XES experiments by means of an FEL operated at given wavelength is that they can provide the spectroscopic energy-resolved response of the system as a function of the intensity of the pulse. As an example, we envisage that by tuning the FEL energy just below an absorption edge, the single-shot XAS (X-ray Absorption Spectroscopy) spectrum can be reconstructed using the Kramers-Heisenberg relationships, as already demonstrated experimentally using 3rd generation facilities. ${ }^{19,20}$ In this way, structural XAS determinations on materials under extreme transient conditions would be available using a single (or a series of) FEL shot of given wavelength.

We present here the design, realization, and characterization of a compact spectrometer for X-ray emission experiments to be performed at FERMI. The instrument has two interchangeable gratings in the $25-800 \mathrm{eV}$ energy range. It can be equipped with different input sections, with/without an entrance slit and with/without an additional relay mirror. These characteristics are achieved in a compact environment resulting in a portable equipment that can be interfaced with different experimental end-stations and covers the whole spectral range of FERMI.

\section{INSTRUMENT DESIGN}

The optical design of the instrument is well established for the use with ultrafast high-order laser harmonics ${ }^{21}$ and FEL radiation. ${ }^{22}$ It consists of a diffraction grating working in grazing incidence and a detector. The grating has a spherical shape with variable line spacing (VLS) along the surfaces, to provide a flat spectral focal plane that is almost perpendicular to the direction of the diffracted light. ${ }^{23}$ The $25-$ $800 \mathrm{eV}$ region is covered by two grazing-incidence VLS spherical gratings (Hitachi cod. 001-0437, $1200 \mathrm{gr} / \mathrm{mm}$ central groove density and cod. 001-0450, $2400 \mathrm{gr} / \mathrm{mm}$ central groove density) that are accommodated on a manually operated linear translation stage to perform the grating selection. The detector is a back-illuminated CCD camera (Princeton Instruments PIXIS-XO 400B, $1340 \times 400$ pixel, $20-\mu \mathrm{m}$ pixel size). Since the length of the focal plane of the $1200 \mathrm{gr} / \mathrm{mm}$ grating is longer than the detector size, the latter is mounted on a motorized linear translation stage and is connected to the grating stage by a bellow, therefore it can be moved so that the central energy can be acquired at the center of the detector plane.

In order to have a flexible instrument that can be accommodated in experimental chambers with different sizes and distances from the target area, three configurations can be realized by interfacing three different input stages to the grating block.

Configuration A, as shown in Fig. 1, has a variable entrance slit that can be manually adjusted from outside. Configuration B, as shown in Fig. 2, has an additional grazingincidence cylindrical mirror acting as a relay section between the slit and the grating. In his way, the distance between the input point and the grating can be increased. This feature is needed in case of large experimental chambers. Both configurations were especially designed for measurements on gas samples. The gas cell (or gas jet) is placed in close proximity of the entrance slit. The instrument is independently pumped by a high-vacuum pumping system and is closed by a shield that aims to maintain the pressure gradient between the inner part and the experimental chamber. Configurations A and B were tailored to the needs of the experimental chambers of the Gas Phase beamline of Elettra and the LDM beamline of FERMI.

Configuration C, shown in Fig. 3, is operated without an entrance slit, since the FEL focal spot on the sample acts as the point-like source of the instrument. It is mainly planned to be used in the EIS-TIMEX chamber of FERMI for measurements on solid targets. The FEL radiation is focused on the sample by a grazing-incidence ellipsoidal mirror, giving a focal spot in the $5-15 \mu \mathrm{m}$ range, which is definitely suitable to act as the source of the spectrometer. Since the entrance arm of the grating is relatively short, i.e., $237 \mathrm{~mm}$, a grazingincidence cylindrical mirror was added to the configuration. It acts as a relay section between the source and the grating and adapts the envelope of the instrument to the size of the experimental chamber that is about $800 \mathrm{~mm}$ in diameter.

The change among the different configurations is a relatively simple operation, since it consists of attaching the proper input stage to the grating block. A photograph of the instrument in Configuration A is shown in Fig. 4. The main instrumental parameters of the spectrometer are summarized in Table I.

The spectral extension of the resolving element, defined as the energy dispersion on the $20-\mu \mathrm{m}$ detector pixel, is shown in Fig. 5. The curve has been inferred from the calibration measurements that will be described below. The efficiency of each component, namely the gratings, the detector and the mirrors, were measured using the calibration facilities available at CNR-IFN Padova (Italy). The global response of the instrument, in terms of counts per input photon, is shown in Fig. 6. 
$0,5 \mathrm{~m}$

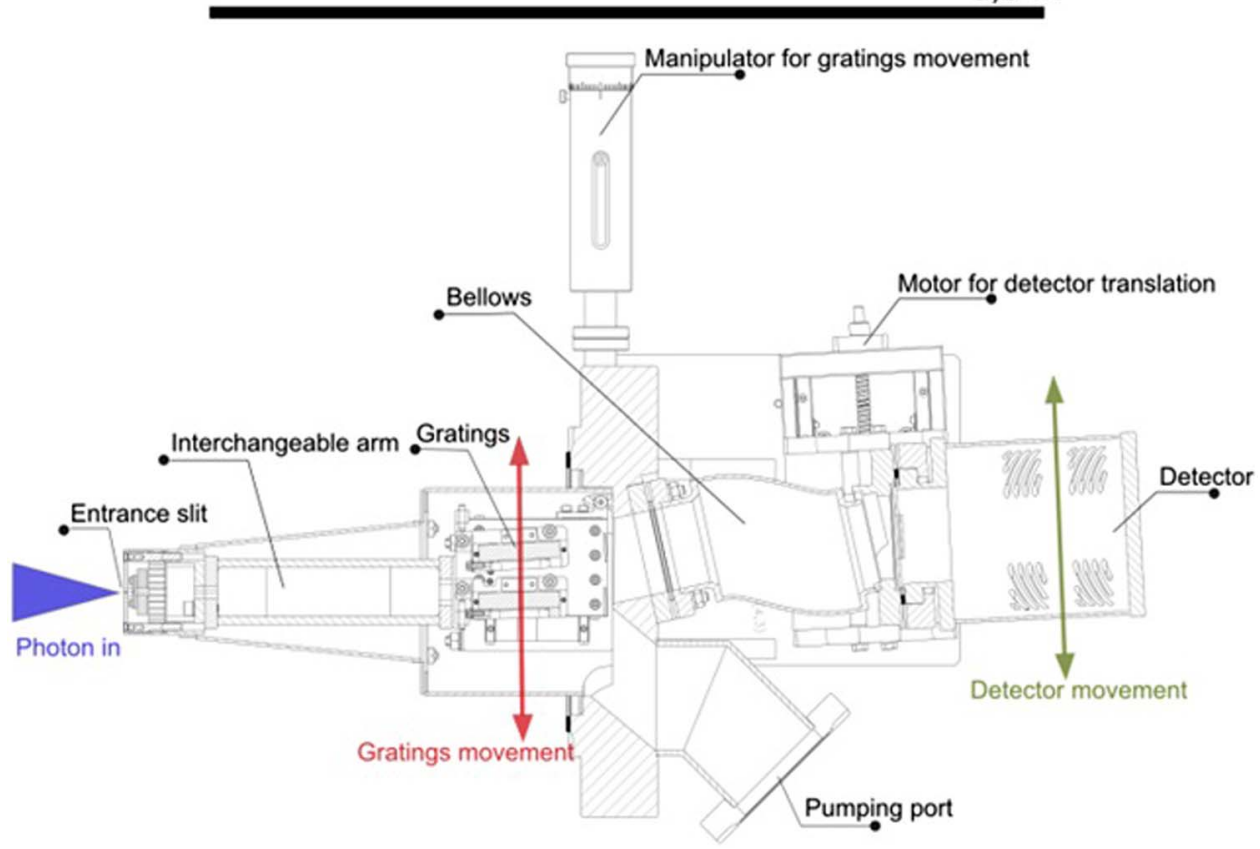

FIG. 1. Spectrometer in configuration A. The detector is mounted on a linear translation stage to be moved along the focal plane and is connected to the instrument flange through a bellow. Gratings can be manually selected from the air side. The input stage has a variable-width entrance slit that can be also actuated from the air side. The instrument is completely shielded to maintain high-vacuum operation and protect it from diffused light. The source-to-grating distance is $237 \mathrm{~mm}$ and the total size of the instrument is about $700 \mathrm{~mm} \times 250 \mathrm{~mm} \times 250 \mathrm{~mm}$.

The collection angle in the direction of the spectral dispersion is limited by the angular acceptance of the gratings, which corresponds to $10 \mathrm{mrad}$ for G1200 and $5 \mathrm{mrad}$ for G2400. In the direction perpendicular to the dispersion, the collection angle is limited by the size of the detector, whose area is $26.8 \mathrm{~mm} \times 8 \mathrm{~mm}$. In case the long detector side $(26.8 \mathrm{~mm})$ is used for acquisition in the spectral direction, the acceptance in the perpendicular direction is in the range 9-17 mrad, where clearly the shorter the source-to-detector distance the higher the acceptance. The acceptance is increased by a factor 3.4 if the detector is rotated $90^{\circ}$, in order to have the short side of the detector $(8 \mathrm{~mm})$ in the dispersion plane. In this case, the spectral range that is simultaneously acquired is correspondingly reduced by a factor 3.4. The values are resumed in Table II. In case a larger CCD detector is adopted (e.g., the Princeton $1340 \times 1340$ format, $20-\mu \mathrm{m}$ pixel size), both the angular acceptance and the extension of the spectral region are maximized.

\section{INSTRUMENT CHARACTERIZATION}

The instrument has been preliminary characterized using the facilities available at CNR-IFN Padova (Italy), consisting of two types of sources emitting in the extreme-ultraviolet and

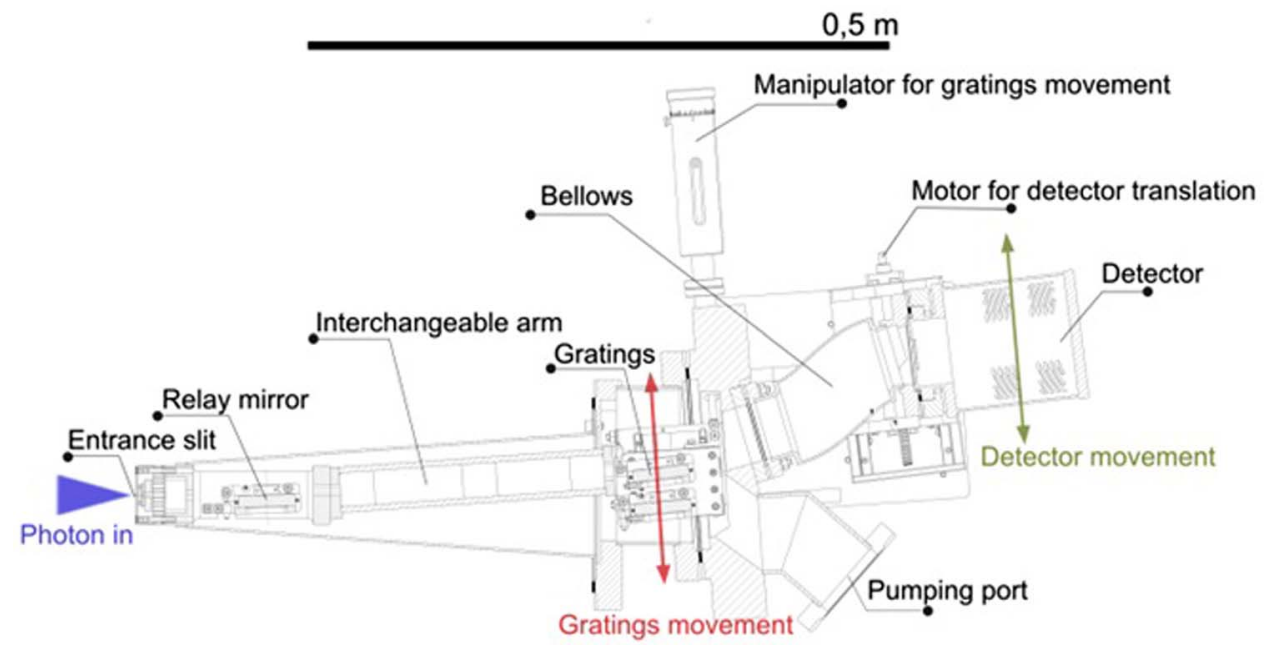

FIG. 2. Spectrometer in configuration B. The input stage has a variable-width entrance slit that can be actuated from the air side and a grazing-incidence cylindrical mirror acting as a relay section, to increase the distance between the source point and the grating. With respect to configuration A, the source-tograting distance has increased to $457 \mathrm{~mm}$ and the total size to $900 \mathrm{~mm} \times 250 \mathrm{~mm} \times 250 \mathrm{~mm}$. 




FIG. 3. Spectrometer in configuration C. The input stage has a grazing-incidence cylindrical mirror acting as a relay section. The entrance-to-grating distance is about $460 \mathrm{~mm}$ and the total size is about $900 \mathrm{~mm} \times 250 \mathrm{~mm} \times 250 \mathrm{~mm}$.

soft X-ray, a grazing-incidence monochromator to increase the spectral purity of the emission and a refocusing section to redirect the monochromatized light to the instrument to be tested. ${ }^{24}$ The available sources are a microfocus electronbombarded source with interchangeable anodes to be used in the 50-400 eV region and a hollow-cathode source to be used in the $25-53 \mathrm{eV}$ region. The instrument was interfaced to the experimental chamber and illuminated with monochromatic light at different energies. All the three configurations (A, B, and C) have been tested with the two gratings. Some of the spectra acquired with the G1200 grating are shown in Fig. 7. Fig. 7(a) shows the spectrum measured with the hollow-cathode lamp filled with He. The width of the spectral lines is about 3 pixels, which is in good agreement with the slit aperture $(100 \mu \mathrm{m})$ and the demagnification factor due to the grating anamorphism. Figure 7(b) shows the emission of the soft-X-ray source with $\mathrm{Al}$ anode at the L-edge at $76 \mathrm{eV}$. Finally, Fig. 7(c) shows the emission of the soft-X-ray source with $\mathrm{C}$ anode. The spectral features are clearly defined and this confirms the good spectral focusing of the instrument.

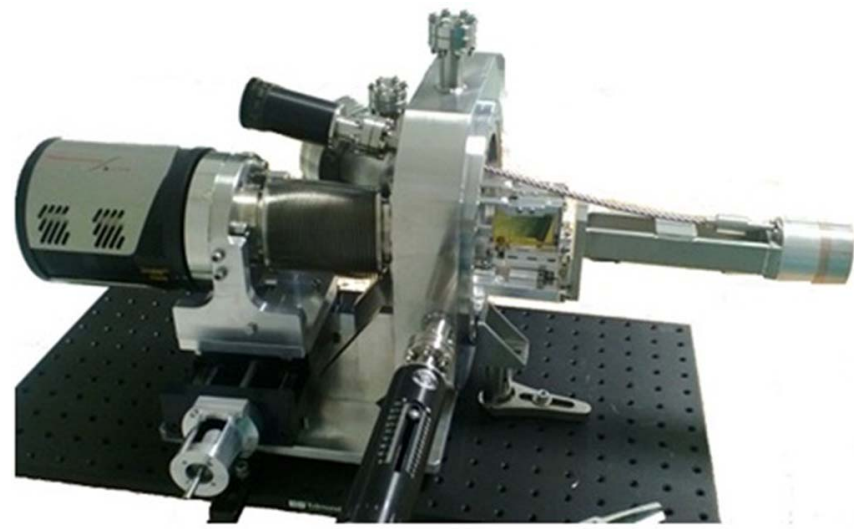

FIG. 4. Picture of the spectrometer in Configuration A. The CCD is mounted on a motorized linear translation stage and it is connected to the grating stage by a bellow. The position of the grating and the slit's aperture can be varied by means of two linear drives placed on the frame of the instrument.
The instrument was further characterized at the Gas Phase beamline ${ }^{25}$ of Elettra synchrotron by measuring fluorescence spectra both from solid and gas targets. The

TABLE I. Instrumental parameters of the spectrometer.

\begin{tabular}{lc}
\hline \hline Grating G1200 & \\
\hline Grating type & Spherical VLS \\
\hline Central groove density & $1200 \mathrm{~mm}^{-1}$ \\
Energy interval & $25-250 \mathrm{eV}$ \\
Incidence angle & $87 \mathrm{deg}$ \\
Entrance arm & $237 \mathrm{~mm}$ \\
Grating-to-detector distance & $235 \mathrm{~mm}$ \\
25-250 eV spectral extension & $58 \mathrm{~mm}$ \\
Grating G2400 & \\
Grating type & Spherical VLS \\
\hline Central groove density & $2400 \mathrm{~mm}-1$ \\
Energy interval & $180-800 \mathrm{eV}$ \\
Incidence angle & $88.7 \mathrm{deg}$ \\
Entrance arm & $237 \mathrm{~mm}$ \\
Grating-to-detector distance & $235 \mathrm{~mm}$ \\
180-800 eV spectral extension & $23 \mathrm{~mm}$ \\
CCD detector & \\
\hline Format & $1340 \times 400$ \\
Pixel size & $20 \times 20 \mu \mathrm{m}$ \\
Detector area & $26.8 \times 8 \mathrm{~mm}$ \\
Configuration A: entrance slit + grating & \\
\hline Slit width & $0.02 \div 1 \mathrm{~mm}$ \\
Slit-to-grating distance & $237 \mathrm{~mm}$ \\
Configuration B: entrance slit + relay mirror $+\mathrm{grating}$ \\
\hline Slit width & $0.02 \div 1 \mathrm{~mm}$ \\
Slit-to-grating distance & $457 \mathrm{~mm}$ \\
Relay mirror input/output arms & $110 \mathrm{~mm}$ \\
Relay mirror incidence angle & $88 \mathrm{deg}$ \\
Configuration C: relay mirror + grating & \\
\hline Source-to-grating distance & $637 \mathrm{~mm}$ \\
Relay mirror input/output arms & $200 \mathrm{~mm}$ \\
Relay mirror incidence angle & $88 \mathrm{deg}$ \\
\hline \hline
\end{tabular}




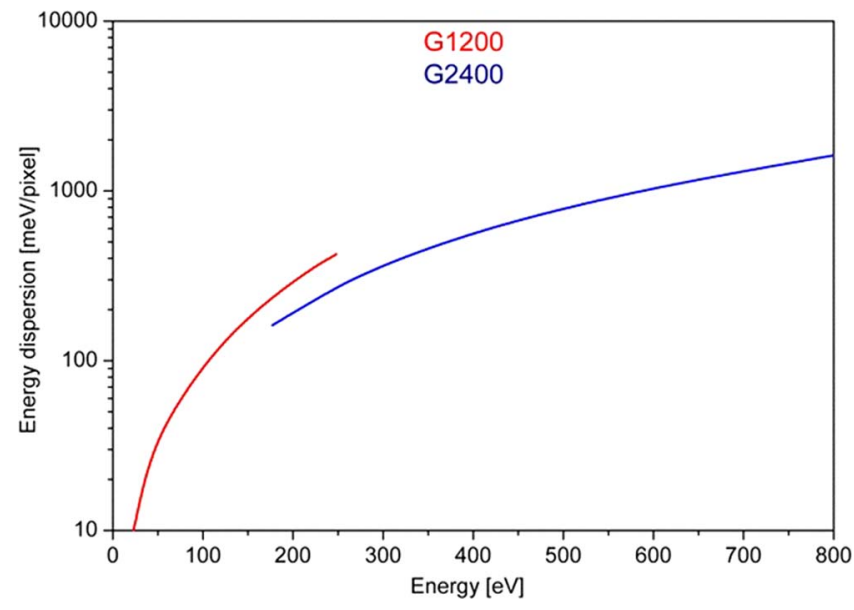

FIG. 5. Spectral extension of the spatial resolving element, defined as the energy dispersion on the $20-\mu \mathrm{m}$ detector pixel.

instrument was set in configuration $\mathrm{A}$ and was mounted perpendicular to the direction of the synchrotron beam (i.e., the angle between the propagation direction of the monochromatized synchrotron beam and the optical axis of the spectrometer was $90^{\circ}$ ) with the entrance slit $10 \mathrm{~mm}$ away from the focal point of the low-energy branch of the Gas Phase beamline. Since the fluorescence spectra that are presented here were acquired with a $200-\mu \mathrm{m}$ slit aperture, the angular acceptance in the spectral direction is limited by the grating acceptance, which is $10 \mathrm{mrad}$ for G1200, and not by the slit-to-sample distance, as this would give an acceptance of 20 mrad. The CCD detector was mounted with its long side parallel to the dispersion plane. The spectrometer was independently pumped through the rear pumping flange to assure high-vacuum operations also with gas targets. The photon flux on the sample was in the $10^{9}-10^{11} \mathrm{ph} / \mathrm{s}$ range, the lower flux being measured above $150 \mathrm{eV}$, where the plane mirror of the branch line has its cutoff in reflectivity.

A gold-coated mirror placed at $45^{\circ}$ incidence angle was initially used as a target to redirect the synchrotron beam toward the spectrometer. Both G1200 and G2400 gratings were

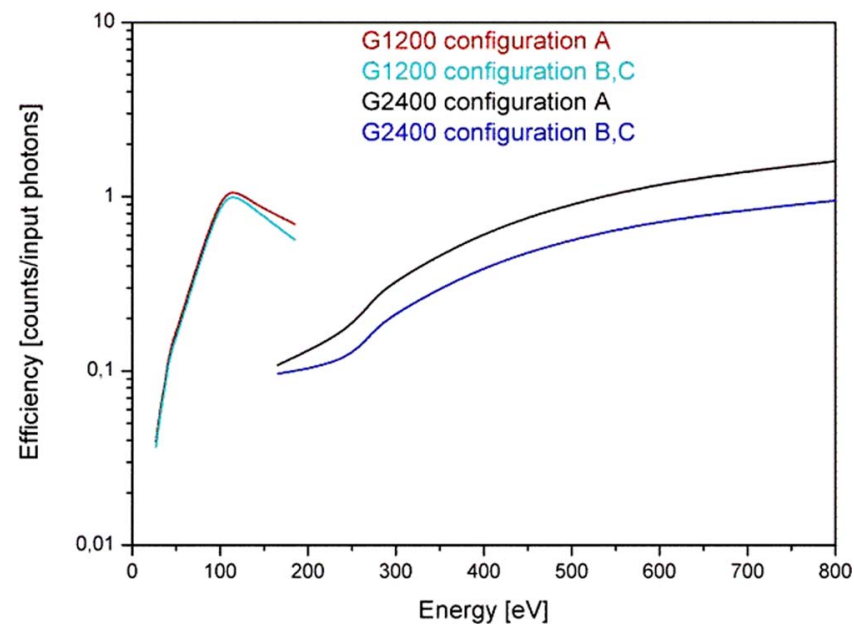

FIG. 6. Global response of the spectrometer, defined as counts on the detector per input photon. It was determined by measuring grating efficiency, detector response and mirror reflectivity (the latter only for configurations B and C).
TABLE II. Angular acceptance and spectral range simultaneously acquired on the spectrometer for the two orientations of the detector.

\begin{tabular}{lc}
\hline \hline Detector spectral extension (long side, 26.8 mm) \\
\hline G1 (three detector positions) & $25-50 \mathrm{eV}$ \\
& $40-112 \mathrm{eV}$ \\
& $60-250 \mathrm{eV}$ \\
& $180-800 \mathrm{eV}$ \\
G2 & $25-30 \mathrm{eV}$ \\
Detector spectral extension (short side, $8 \mathrm{~mm}$ ) & $40-51 \mathrm{eV}$ \\
G1 (some examples) & $100-160 \mathrm{eV}$ \\
\multicolumn{2}{c}{$140-250 \mathrm{eV}$} \\
G2 (three detector positions) & $180-270 \mathrm{eV}$ \\
\multicolumn{2}{c}{$330-600 \mathrm{eV}$} \\
Acceptance angle, spectral dispersion plane & $400-800 \mathrm{eV}$ \\
G1200 & $10 \mathrm{mrad}$ \\
G2400 & $5 \mathrm{mrad}$ \\
Acceptance angle, plane $\perp$ to the dispersion & \\
\hline Detector short side $\perp$ to the dispersion & $17 \mathrm{mrad}$ \\
Configuration A & $12 \mathrm{mrad}$ \\
Configuration B & $9 \mathrm{mrad}$ \\
Configuration C & $57 \mathrm{mrad}$ \\
Detector long side $\perp$ to the dispersion & $40 \mathrm{mrad}$ \\
Configuration A & $30 \mathrm{mrad}$ \\
Configuration B & \\
Configuration C & \\
\hline \hline
\end{tabular}

calibrated in energy by acquiring the signal reflected by the mirror and entering into the spectrometer in the $250-25 \mathrm{eV}$ energy region. The synchrotron bandwidth was narrower than the spectral extension of the CCD pixel. We measured sharp spectral lines that were about 3 pixels wide when using a 100$\mu \mathrm{m}$ slit, again confirming the good spectral focusing of the configuration as already mentioned. Furthermore, we have acquired several spectra at different energies that confirm the dispersion curve as shown in Fig. 5. Although the mirror was set at $45^{\circ}$ incidence angle and a relatively low photon flux, especially above $150 \mathrm{eV}$, was expected, the reflected signal was acquired in $0.1-1 \mathrm{~s}$. At $250 \mathrm{eV}$, where the photon flux is expected to be definitely lower than $10^{9} \mathrm{ph} / \mathrm{s}$ and the sample reflectivity lower than $10^{-4}$, the calibration spectra were acquired in $1 \mathrm{~s}$. This confirms the good instrumental sensitivity.

\section{A. Fluorescence on solid samples}

The characterization of the spectrometer as an instrument for photon in-photon out experiments was initially performed by investigating fluorescence from solid samples. Measurements were performed on a piece of silicon wafer and on pellets of boron and boron nitride, pressed from powder. All the samples were positioned on the focus of the branch line (focus size $\approx 200 \mu \mathrm{m}$ ) at an incidence angle in the range $60^{\circ}-$ $80^{\circ}$, in order to have the elastic contribution definitely out from the acceptance of the spectrometer and to collect only the isotropic inelastic portion.

A fluorescence spectrum of $\mathrm{Si}$ taken at the $\mathrm{Si} L$ edge is shown in Fig. 8. The spectrum resembles and is in overall agreement with previously published data on crystalline Si films. ${ }^{26}$ The peaks near 89 and $92 \mathrm{eV}$ originate from 



(b)

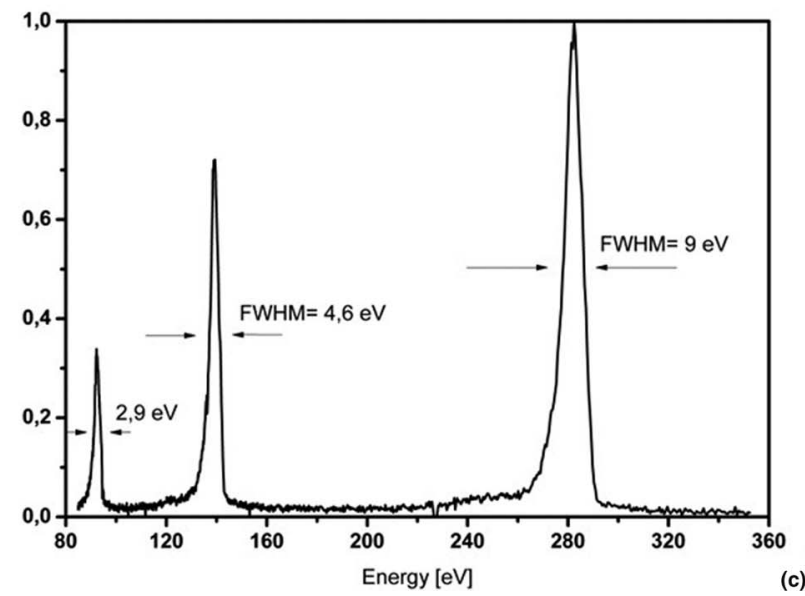

FIG. 7. Spectra obtained with the G1200 grating in the laboratory characterization: (a) hollow-cathode lamp filled with $\mathrm{He}$; (b) microfocus source with $\mathrm{Al}$ anode; and (c) microfocus source with $\mathrm{C}$ anode. The spectra have been taken with $100-\mu \mathrm{m}$ entrance slit. All the three configurations have been measured and give almost the same spectra.

nonbonding $s$ states and $s p$-hybridized states, respectively. B and $\mathrm{BN}$ fluorescence spectra taken at the B K edge are shown in Fig. 9. The emission peaks, characteristic of the $\mathrm{K} \alpha$ transition in $\mathrm{B}$, are observed at $180 \mathrm{eV}$ for both $\mathrm{B}$ and $\mathrm{BN}$, in agreement with data already reported in the literature. ${ }^{27}$

\section{B. Fluorescence on gas-phase}

The spectrometer was characterized also as an instrument to measure fluorescence from gas-phase targets. A suitable

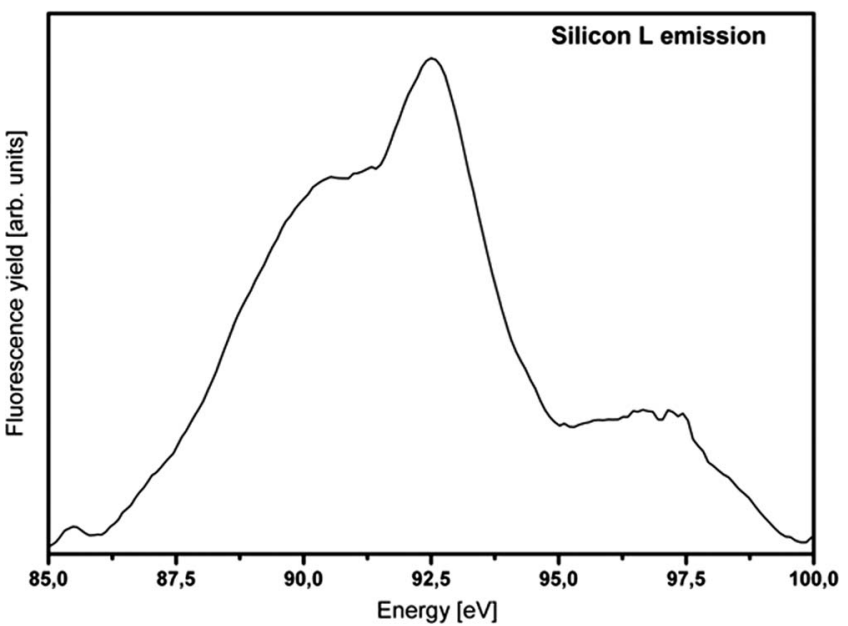

FIG. 8. Si L emission fluorescence spectrum for crystalline Si sample measured with the G1200 grating. The exciting photon energy is $100.5 \mathrm{eV}$ and the acquisition time $15 \mathrm{~min}$.

gas cell was mounted close to the entrance slit of the spectrometer, having entrance and exit holes for the synchrotron beam and a lateral slit (120- $\mu \mathrm{m}$ wide and 5-mm long) parallel to the entrance slit of the spectrometer, to maximize the throughput of the fluorescence collected by the instrument. Fluorescence spectra of helium and krypton were acquired. When the gas was injected in the cell, the pressure in the experimental chamber was stabilized to $\approx 1 \times 10^{-3} \mathrm{mbar}$ and the pressure inside the gas cell was estimated to be in the several- $10^{-1}$ mbar range. The entrance slit was kept at $100 \mu \mathrm{m}$. The instrument could be operated at a pressure of $\approx 10^{-4}$ mbar thanks to the differential vacuum stage provided by the slit and the independent pumping system.

As a test run with $\mathrm{He}$, we measured spectra emitted when the exciting photon energy was fixed either to the $(3,0)$ resonance at $64.118 \mathrm{eV}$ or to the $(4,-1)$ resonance at $64.133 \mathrm{eV}$. The beamline monochromator was set to give an output bandwidth of $11 \mathrm{meV}$ at $64 \mathrm{eV}$. The emission lines observed with

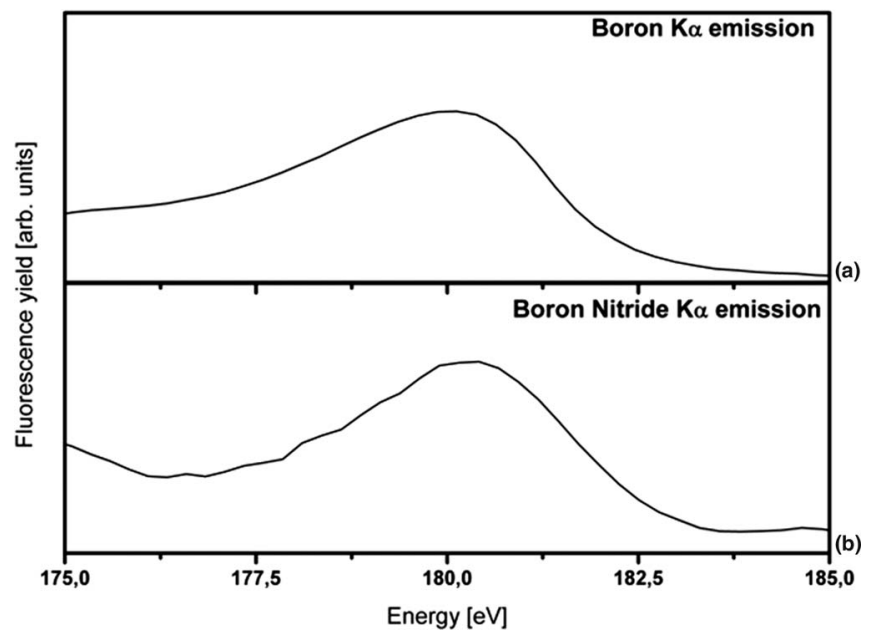

FIG. 9. B (a) and BN (b) K $\alpha$ emission fluorescence spectra measured with the G1200 grating. In case of B, the exciting photon energy is $220 \mathrm{eV}$ and the acquisition time $15 \mathrm{~min}$. In case of BN, the exciting photon energy is $200 \mathrm{eV}$ and the acquisition time $90 \mathrm{~min}$. The G1200 grating was used. 


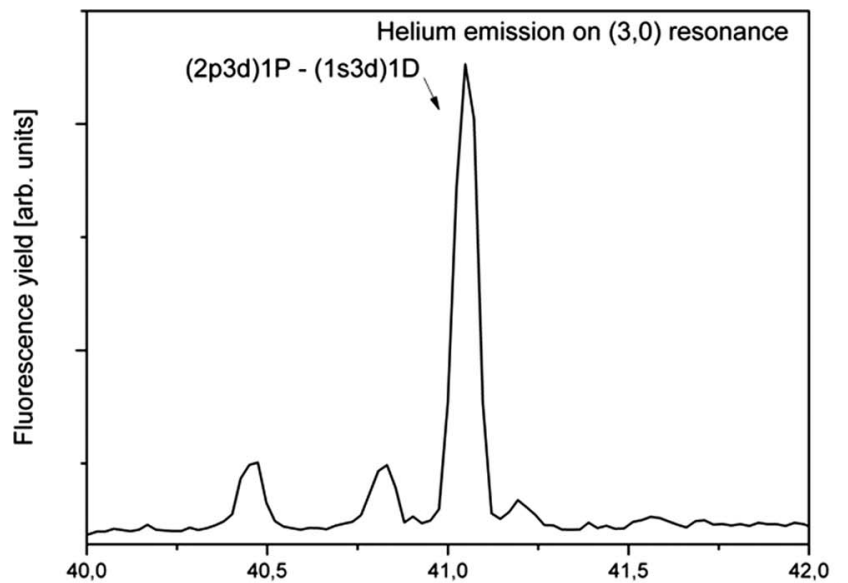

(a)

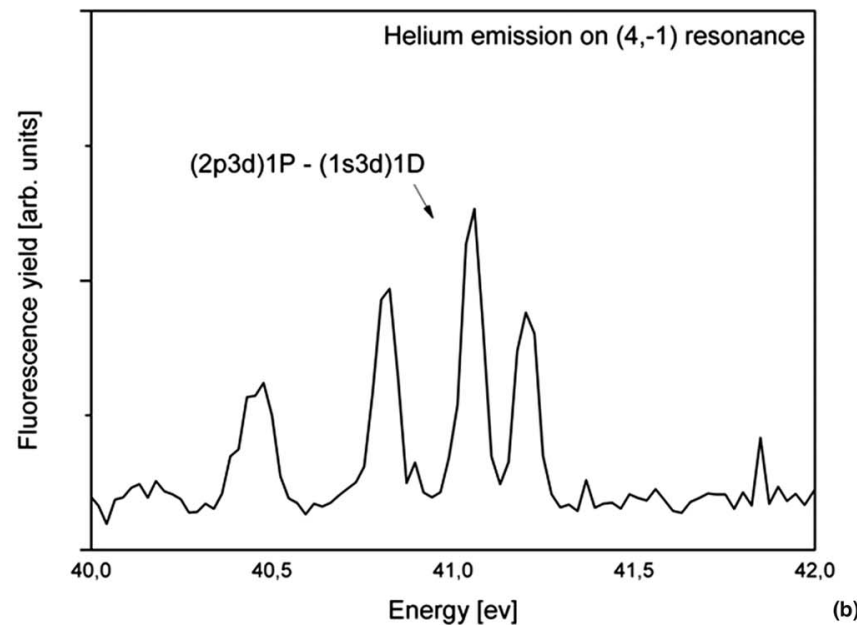

FIG. 10. He fluorescence spectra from the doubly excited states of helium below the $\mathrm{N}=2$ ionization threshold measured with the G1200 grating. The acquisition time was $10 \mathrm{~min}$ and the gas cell pressure 0.8 mbar. (a) $(3,0)$ resonance at $64.114 \mathrm{eV}$ and (b) $(4,-1)$ resonance at $64.133 \mathrm{eV}$.

the spectrometer in the different experimental conditions are shown in Fig. 10 and are in agreement with the data already available. ${ }^{28}$ When exciting at $64.118 \mathrm{eV}$, a peaked photon emission due to the $(2 \mathrm{p} 3 \mathrm{~d})^{1} \mathrm{P}-(1 \mathrm{~s} 3 \mathrm{~d})^{1} \mathrm{D}$ transition is measured, whereas the emission at $40.81 \mathrm{eV}$ is due to Helium pho-

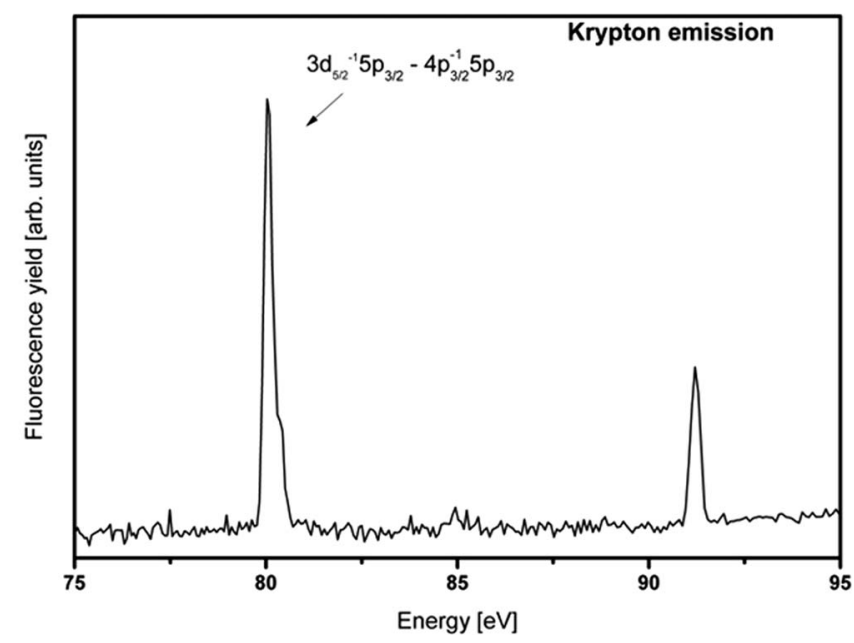

FIG. 11. Krypton fluorescence spectrum with $91.2 \mathrm{eV}$ exciting photon energy measured with the G1200 grating. The acquisition time was $15 \mathrm{~min}$. toionization from higher-orders synchrotron light transmitted through the beamline monochromator. The emission spectrum changes when different resonances of $\mathrm{He}$ are populated, as the case of the $(4,-1)$ resonance at $64.133 \mathrm{eV}$. Note that the acquired spectra exhibit a higher signal-to-noise ratio than those reported in the literature.

Fluorescence emission from $\mathrm{Kr}$ excited at the $M_{4,5}$ edge was also measured. Fig. 11 shows the spectrum with exciting photon energy at the $\mathrm{Kr} 3 d_{5 / 2} \rightarrow 5 p$ resonance $(91.2 \mathrm{eV})$. The $\mathrm{Kr} 3 d_{\frac{5}{2}}^{-1} 5 p_{3 / 2}(J=1) \rightarrow 4 p_{\frac{3}{2}}^{-1} 5 p_{\frac{3}{2}}(J=0,2)$ transitions are clearly visible around $80 \mathrm{eV}$, while the elastic peak can be seen at $91.2 \mathrm{eV}$.

\section{CONCLUSION}

We have presented the design and characterization of a portable and compact photon spectrometer to be used for photon in-photon out experiments at synchrotron and FEL sources. The instrument was fully characterized by measuring fluorescence spectra both from solid and gas targets. The collected spectra are in full agreement with data reported in the literature, in particular the fluorescence spectra from gas targets have a higher signal-to-noise ratio than the data reported in the literature, opening the way to the use of the spectrometer for specific gas targets where experimental data are missing.

Besides the use in synchrotron beamlines, the instrument is specially designed to be interfaced on the LDM and EISTIMEX beamlines at FERMI, where research activities in the field of atomic and molecular physics and in condensed matter are conducted. The apparatus may be also used for timeresolved pump-probe experiments in the strong-optical-field regime, that is obtained by combining the FEL radiation with the ultrashort 780-nm laser that is also available at FERMI. Finally, it is also possible to mount it at the end of the beamlines with its own support as a diagnostic tool complementary to the high-resolution spectrometer presently available at FERMI to monitor the FEL spectral emission..$^{29}$ The spectrometer was already employed at FERMI to measure single-shot FEL-2 emission in the $300-800 \mathrm{eV}$ region.

\section{ACKNOWLEDGMENTS}

The authors would like to thank Dr. Carlo Callegari (Elettra) and Dr. Monica de Simone (CNR-IOM) for their support to our project and in particular for their help during the commissioning of the instrument at the Gas Phase beamline. Dr. Emiliano Principi (Elettra) is also acknowledged for support in defining the interface of the instrument to EIS -TIMEX at FERMI.

This work was supported by the project "Single-shot X-ray emission-spectroscopy experiments," funded by the Italian Ministry for Education and Research as In-Kind Project for the EuroFEL consortium.

${ }^{1}$ U. Bergmann and P. Glatzel, Photosynth. Res. 102, 255 (2009).

${ }^{2}$ J. Yano and V. K. Yachandra, Photosynth. Res. 102, 241 (2009).

${ }^{3}$ W. Schuelke, Electron Dynamics by Inelastic X-Ray Scattering (Oxford University Press, Oxford, 2007). 
${ }^{4}$ F. De Groot and A. Kotani, Core Level Spectroscopy of Solids (CRC Press, 2008).

${ }^{5}$ M. Beye, F. Sorgenfrei, W. F. Schlotter, W. Wurth, and A. Föhlisch, Proc. Natl. Acad. Sci. U.S.A. 107, 16772 (2010)

${ }^{6}$ M. Altarelli, Eur. Phys. J.: Spec. Top. 208, 351 (2012).

${ }^{7}$ D. Zhu, M. Cammarata, J. M. Feldkamp, D. M. Fritz, J. B. Hastings, S. Lee, H. T. Lemke, A. R. James, L. Turner, and Y. Feng, Appl. Phys. Lett. 101, 034103 (2012).

${ }^{8}$ K. Kunnus, I. Rajkovic, S. Schreck, W. Quevedo, S. Eckert, M. Beye, E. Suljoti, C. Weniger, C. Kalus, S. Grübel, M. Scholz, D. Nordlund, W. Zhang, R. W. Hartsock, K. J. Gaffney, W. F. Schlotter, J. J. Turner, B. Kennedy, F. Hennies, S. Techert, P. Wernet, and A. Föhlisch, Rev. Sci. Instrum. 83, 123109 (2012).

${ }^{9}$ T. Katayama, T. Anniyev, M. Beye, R. Coffee, M. Dell'Angela, A. Föhlisch, J. Gladh, S. Kaya, O. Krupin, A. Nilsson, D. Nordlund, W. F. Schlotter, J. A. Sellberg, F. Sorgenfrei, J. J. Turner, W. Wurth, H. Öström, and H. Ogasawara, J. Electron Spectrosc. Relat. Phenom. 187, 9-14 (2013).

${ }^{10}$ V. Lyamayev, Y. Ovcharenko, R. Katzy, M. Devetta, L. Bruder, A. LaForge, M. Mudrich, U. Person, F. Stienkemeier, M. Krikunova, T. Möller, P. Piseri, L. Avaldi, M. Coreno, P. O'Keeffe, P. Bolognesi, M. Alagia, A. Kivimäki, M. Di Fraia, N. B. Brauer, M. Drabbels, T. Mazza, S. Stranges, P. Finetti, C. Grazioli, O. Plekan, R. Richter, K. C. Prince, and C. Callegari, J. Phys. B 46, 164007 (2013).

${ }^{11}$ A. Di Cicco, F. Bencivenga, A. Battistoni, D. Cocco, R. Cucini, F. D’Amico, S. Di Fonzo, A. Filipponi, A. Gessini, E. Giangrisostomi, R. Gunnella, C. Masciovecchio, E. Principi, and C. Svetina, Proc. SPIE 8077, 807704 (2011)

${ }^{12}$ E. Allaria, R. Appio, L. Badano, W. A. Barletta, S. Bassanese, S. G. Biedron, A. Borga, E. Busetto, D. Castronovo, P. Cinquegrana, S. Cleva, D. Cocco, M. Cornacchia, P. Craievich, I. Cudin, G. D’Auria, M. Dal Forno, M. B. Danailov, R. De Monte, G. De Ninno, P. Delgiusto, A. Demidovich, S. Di Mitri, B. Diviacco, A. Fabris, R. Fabris, W. Fawley, M. Ferianis, E Ferrari, S. Ferry, L. Froehlich, P. Furlan, G. Gaio, F. Gelmetti, L. Giannessi, M. Giannini, R. Gobessi, R. Ivanov, E. Karantzoulis, M. Lonza, A Lutman, B. Mahieu, M. Milloch, S. V. Milton, M. Musardo, I. Nikolov, S. Noe, F. Parmigiani, G. Penco, M. Petronio, L. Pivetta, M. Predonzani, F. Rossi, L. Rumiz, A. Salom, C. Scafuri, C. Serpico, P. Sigalotti, S. Spampinati, C. Spezzani, M. Svandrlik, C. Svetina, S. Tazzari, M. Trovo, R. Umer,
A. Vascotto, M. Veronese, R. Visintini, M. Zaccaria, D. Zangrando, and M. Zangrando, Nat. Photonics 6, 699 (2012).

${ }^{13}$ W. Zhang, V. Carravetta, O. Plekan, V. Feyer, R. Richter, M. Coreno, and K. C. Prince, J. Chem. Phys. 131, 035103 (2009).

${ }^{14}$ M. Coreno, M. de Simone, R. Coates, M. S. Denning, R. G. Denning, J. C. Green, C. Hunston, N. Kaltsoyannis, and A. Sella, Organometallics 29, 4752 (2010)

${ }^{15}$ L. Ravagnan, T. Mazza, G. Bongiorno, M. Devetta, M. Amati, P. Milani, P. Piseri, M. Coreno, C. Lenardi, F. Evangelista, and P. Rudolf, Chem. Commun. 47, 2952 (2011)

${ }^{16} \mathrm{H}$. Wabnitz et al., Nature (London) 420, 482 (2002).

${ }^{17}$ P. Glatzel, M. Sikora, and M. Fernandez-Garcia, Eur. Phys. J.: Spec. Top. 169, 207 (2009).

${ }^{18}$ A. Di Cicco, F. D’Amico, G. Zgrablic, E. Principi, R. Gunnella, F. Bencivenga, C. Svetina, C. Masciovecchio, F. Parmigiani, and A. Filipponi, J. Non-Cryst. Solids 357, 2641 (2011).

${ }^{19}$ H. Hayashi, R. Takeda, Y. Udagawa, T. Nakamura, H. Miyagawa, H. Shoji, S. Nanao, and N. Kawamura, Phys. Rev. B 68, 045122 (2003).

${ }^{20}$ J. J. Kas, J. J. Rehr, J. A. Soininen, and P. Glatzel, Phys. Rev. B 83, 235114 (2011).

${ }^{21}$ L. Poletto, G. Tondello, and P. Villoresi, Rev. Sci. Instrum. 72, 2868 (2001).

${ }^{22}$ F. Frassetto, S. Coraggia, S. Dziarzhytski, N. Gerasimova, and L. Poletto, J. Synchrotron Radiat. 19, 596 (2012).

${ }^{23}$ T. Kita, T. Harada, N. Nakano, and H. Kuroda, Appl. Opt. 22, 512 (1983).

${ }^{24}$ L. Poletto, A. Boscolo, and G. Tondello, Proc. SPIE 3764, 94-102 (1999).

${ }^{25}$ R. R. Blyth, R. Delaunay, M. Zitnik, J. Krempasky, R. Krempaska, J. Slezak, K. C. Prince, R. Richter, M. Vondracek, R. Camilloni, L. Avaldi, M. Coreno, G. Stefani, C. Furlani, M. de Simone, S. Stranges, and M.-Y. Adam, J. Electron Spectrosc. Relat. Phenom. 101-103, 959 (1999).

${ }^{26}$ J. A. Carlisle, A. Chaiken, R. P. Michel, L. J. Terminello, J. J. Jia, T. A. Callcott, and D. L. Ederer, Phys. Rev. B 53, R8824 (1996).

${ }^{27}$ Y. Muramatsu, M. Oshima, and H. Kato, Phys. Rev. Lett. 71, 448 (1993).

${ }^{28}$ K.-H. Schartner, B. Zimmermann, S. Kammer, S. Mickat, H. Schmoranzer, A. Ehresmann, H. Liebel, R. Follath, and G. Reichardt, Phys. Rev. A 64, 040501 (2001).

${ }^{29}$ M. Zangrando, A. Abrami, D. Bacescu, I. Cudin, C. Fava, F. Frassetto, A. Galimberti, R. Godnig, D. Giuressi, L. Poletto, L. Rumiz, R. Sergo, C. Svetina, and D. Cocco, Rev. Sci. Instrum. 80, 113110 (2009). 